\title{
Tubeless Ureterorenoscopy. A Dangerous Adventure or "Fresh Wind" Relied on Skills, Technique and New Technology?
}

\author{
Itay M. Sabler ${ }^{1 *}$, Ioanis Katafigiotis ${ }^{2,3}$ and Mordechai Duvdevani ${ }^{3}$ \\ ${ }^{1}$ Department of Urology, Endourology and Lithotripsy Unit, Shamir (Assaf Harofeh) Medical Center, Affiliated with Sackler school of Medicine, Tel Aviv University, \\ Israel
}

${ }^{2}$ Athens Stone Clinic, Athens, Greece

${ }^{3}$ Department of Urology, Hadassah Hebrew University Medical Center, Jerusalem, Israel

*Corresponding author: Itay M Sabler, Department of Urology, Endourology and Lithotripsy Unit, Tel Aviv University, Shamir (Assaf Harofeh) Medical Center, Affiliated with Sackler school of Medicine, Israel.

Received Date: April 29, 2019

Published Date: June 06, 2019

\begin{abstract}
Upper urinary tract endoscopic stone treatment includes intracorporeal lithotripsy, usually using Holmium laser fiber, and temporary drainage of the upper urinary tract postoperatively. Almost absolute endourologic routine is to leave Double-J stent (DJS) for several weeks or ureteral catheter attached to urethral catheter for 24-72 hours. The reason for that is to prevent postoperative pain and infection due to local edema at the ureteral orifice, and upper urinary tract obstruction. On the other hand, postoperative tubing is known to cause lower urinary tract symptoms (LUTS), abdominal and flank pain. Postoperative tubing may cause additional emergency department visits, analgesics use, preliminary interventions and in case of DJS, usually demands invasive procedure, sometimes under general anesthesia in order to retrieve the stent after predetermined period of carriage. At the end of Tubeless Ureterorenoscopy (URS) for treatment of kidney and ureteral stones - no drainage left. Patient comfort advantages of tubeless approach are obvious, but fear of obstruction precluded urologist all over the world from leaving upper tracts undrained for decades. These days, technological achievements enable endourologists to use miniaturized flexible or semirigid ureteroscopes and novel high-power laser machines, minimizing upper urinary tract damage during the procedure and promoting a very effective stone dusting never seen before. These factors permit, in selected cases, to avoid postoperative tubing, reduce LUTS, and shorten hospitalization period facilitating ambulatory nature of the procedure with overall decreasing costs.
\end{abstract}

\section{Conclusion}

A tubeless approach is safe in properly selected uncomplicated cases. The postoperative period is at least the same as in drained patients, avoiding long term postoperative stent related symptoms. More RCT are needed to point the place for safe tubeless endourologic procedures.

Keywords: Ureterorenoscopy; Tubeless; Upper urinary tract drainage; Kidney stones; Ureteral stones; Lithotripsy

Abbreviations: URS- Ureterorenoscopy, LUTS - Lower Urinary Tract Symptoms; DJS - Double J Stent; UC - Ureteral Catheter; SWL - Shock Wave Lithotripsy; RCT - Randomized Controlled Trial

\section{Introduction}

Dramatic financial investments and technologic advancement of Endourology during the last few decades are well known and appreciated. This fact locates it at a spearhead of modern urology and medicine. New technologies and techniques are available, new approaches are developed. Tubeless upper urinary tract procedures are not new, and the necessity of postoperative drainage has been debated for decades [1]. A ureteral catheter (UC) and Double-J stent (DJS) are used routinely all other the world to avoid postoperative pain, prevent infections and strictures and facilitate fragments expulsion. The concern is obstruction secondary to intramural ureteral edema [2]. Despite that, foreign bodies left postoperatively cause symptoms that include LUTS, flank or abdominal pain and are recognized and managed at immediate postoperative and longterm periods, and sometimes demand urgent interventions. The extraction of the DJS is done by local or general anesthesia by an invasive cystoscopy. It's been shown that postoperative drainage doesn't carry any significant advantages over the tubeless procedure in stone-free, infections, morbidity or analgesia requirements after uncomplicated URS $[3,4]$. 


\section{Discussion}

In our series of above 500 cases of endoscopic upper urinary tract stones treatment, about $40 \%$ of patients were left tubeless and had the same analgesic demands at an immediate postoperative period as those drained by UC, which necessitates urethral catheter attachment causing additional lower tract symptoms. Further on, it was shown that the stone burden affected significantly the decision of postoperative drainage type and tubeless were preferably left cases with a relatively low one. In our practice, stone volume, density, and preoperative severe hydronephrosis affected the decision to drain the upper tract significantly. More than that, higher stone volume and higher stone density produce a considerable volume of bigger fragments, and postoperative drainage of the upper urinary tract was necessary. In other cases, according to our data, a stent or a UC may be omitted [5].

We use a sheath less dusting technique in our routine URS practice for stone management. Access sheaths, widely used in endourologic practice, enable better visualization during the procedure and prevent elevation of intrapelvic intraoperative pressure, one of the possible causes of postoperative infectious complications. On the other hand, tiny ureteral lumen, is unavoidably traumatized by access sheath insertion and always necessitates postoperative drainage to let edema to subside and ureteral urothelium to heal. On the contrary, the sheath less approach using miniaturized, flexible ureteroscope in the absence of traumatic ureteral engagement, enables safe tubeless procedures.

In order to understand better the essence behind the tubeless approach, we should remember Shock Wave Lithotripsy (SWL) treatment [6]. No tubing is usually needed or used after the procedure for kidney stones of less than or equal to $10 \mathrm{~mm}$ in length or small ureteral stones treatment. No doubt, SWL has less ability to control produced stone particles size than endoscopic approach, thus having increased risk of obstruction further on, when those fragment rush through the ureter to be expelled into the bladder.

Another important factor is, as was mentioned before, tremendous technological achievements of the last decade and among them the invention of powerful lasers able to fast and efficiently break the stones intracorporeally. One of the latest examples of the development of laser technology is Lumenis Pulse and Versa Pulse ${ }^{\circledR}$ Power Suite ${ }^{\mathrm{TM}}$ using MosesTM technology. The power is delivered through laser fibers at 120Watt max. The repetition rates up to 80 Hertz and low energy pulses (0.2-0.6 Joules) enable efficient and fast dusting of renal and ureteral stones. The conclusions of the preclinical comparison trial were that MosesTM showed more efficient laser lithotripsy and significantly reduced stone retropulsion resulting in significantly shorter operation time and higher safety [7].

Such powerful devices produce "dust" so small particles that their passive expulsion, later can be asymptomatic even in the postoperatively undrained patient. Despite that, the stone burden size treated by the dusting technique should be carefully analyzed by the endourologist. High stone volume produces a big amount of dust and precaution should be undertaken by postoperative DJS draining, for dilatation of the upper tract and avoiding the stone street of dust particles to obstruct the kidney.

Some examples of brief literature review, that revealed quite sparse data, are: Byrne et al showed no difference in LUTS between the stented and tubeless groups at POD 1, but symptoms were significantly reduced further on in tubeless cases [8]. Denstedt et al stated that routine stenting is not mandatory after uncomplicated URS and noted significantly more symptoms in stented patients without complications or stone-free difference [9]. Stenting failed to improve stone-free and, instead, caused additional complications in Wang et al combined data from 22 RCT, but worth mentioning, that stenting prevented re-hospitalization [10].

No doubt, careful patient selection, endoscopic technique, and technological achievements are three main parameters that enable safe tubeless URS. In order to conclude the place, and appoint indications and cut off for tubeless approach there is a need for RCT directed to answer still existing questions about Stone burden limits? Patient and stone characteristics? Re-treatment and re-hospitalization rates? Stone free? Complications? Costs and operation time comparison? Additionally, would be interesting to divide tubeless and stented patients to stone volume subgroups in a prospective randomized trial with long term follow up.

\section{Conclusion}

There is no consensus or guidelines that select cases suitable for a safe tubeless approach. Short-term drainage with UC does not change the postoperative course and, therefore, its use postoperatively is questionable. The tubeless approach might be safe and justified, especially from a symptomatic point of view, in properly selected cases. Tubeless procedures might improve immediate and mid-term postoperative periods with equal stonefree rates and enable an outpatient approach to the upper urinary tract stones treatment. There is a need for properly constructed RCT to discover the pro's and con's and develop an intraoperative decision-making process relied on evidence-based data.

\section{Acknowledgements}

Katafigiotis I-substantial contribution to acquisition, analysis and interpretation of data, Duvdevani M-substantial contributions to acquisition, analysis and interpretation of data.

\section{Conflict of Interest}

No conflicts of interest.

\section{References}

1. Gunlusoy B, Degirmenci T, Arslan M, Kozacýoglu Z, Minareci S, et al. (2008) Is ureteral catheterization necessary after ureteroscopic lithotripsy for uncomplicated upper ureteral stones? J Endourol 22(8): $1645-1648$.

2. Gettman MT, Segura JW (2005) Management of ureteric stones: Issues and controversies. BJU Int 95(Suppl 2): 85-93.

3. Gunlusoy B, Degirmenci T, Arslan M, Kozacýoglu Z, Minareci S, et al. (2008) Is ureteral catheterization necessary after ureteroscopic lithotripsy for uncomplicated upper ureteral stones? J Endourol 22(8): 1645-1648. 
4. Makarov DV, Trock BJ, Allaf ME, Matlaga BR (2008) The effect of ureteral stent placement on post-ureteroscopy complication: A meta-analysis. Urology 71(5): 796-800.

5. Sabler IM, Isid A, Katafigiotis I, Lorber A, Latke A, et al. (2018) Does retrograde treatment of upper urinary tract stones necessitate postoperative upper urinary tract drainage? Conclusions from more than 500 single center consecutive cases. J Endourol 32(6): 477-481.

6. Shen P, Jiang M, Yang J, Li X, Li Y, et al. (2011) Use of ureteral stent in extracorporeal shock wave lithotripsy for upper urinary calculi: A systematic review and meta-analysis. J Urol 186(4): 1328-1335.

7. Elhilali M, Badaan S, Ibrahim A, Andonian S (2017) Use of Moses Pulse Modulation Technology to Improve Holmium Laser Lithotripsy Outcomes: A preclinical study. J Urol 31(6): 598-604.
8. Byrne RR, Auge BK, Kourambas J, Munver R, Delvecchio F, et al. (2002) Routine ureteral stenting is not necessary after ureteroscopy and ureteropyeloscopy: A randomized trial. J Endourol 16(1): 9-13

9. Denstedt JD, Wollin TA, Sofer M, Nott L, Weir M, et al. (2001) A prospective randomized controlled trial comparing nonstented versus stented ureteroscopic lithotripsy. J Urol 165(5): 1419-1422.

10. Wang H, Man L, Li G, Huang G, Liu N, et al. (2017) Meta-analysis of stenting versus non-stenting for the treatment of ureteral stones. PLoS One 12(1): e0167670. 